of Ireland for apparatus used in this investigation, and Messrs. John Player and Sons, Dublin, for a generous gift of 'Cellophane'.

Department of Physics,

$$
\text { E. F. FAHX }
$$

Department of Anatomy,

M. A. MacConaili

University College, Cork.

1 MacConaill, M. A., Dental. Rec., 75 (April 1952).

\section{Threshold Potential for the Low-Frequency Electric Discharge in Air}

STUDIES of the threshold potential ${ }^{1}, V_{m}$, for low. frequency $(50$ c. $/ \mathrm{s}$.) discharges in atmospheric air dried over phosphorus pentoxide were made over a range of pressure, $p$, from 1 to $75 \mathrm{~mm}$. of mercury, using cylindrical glass tubes with plane parallel glass ends as electrodes. $\quad V_{m}$ was indicated by a sudden rise in the current flowing through the discharge tube, the initiation of a glow characteristic of the gas, and the appearance of high-frequency pulses on the current waveform.

Fig. 1 shows the variation of $V_{m}$ (a.c. peak value) with the product of pressure and inter-electrode distances, $\bar{d}$ in cm., (i) for hard 'Pyrex' glass electrodes and (ii) for soft soda glass electrodes. For comparison, values of the sparking potential, $V_{s}$, are also shown (iii) for brass electrodes under $50 \mathrm{c}$./s. a.c. excitation, and (iv) for mercury-contaminated copper electrodes under d.c. excitation recently reported by Llewellyn Jones and Davies ${ }^{2}$.

It is seen that for a given value of $p \times d, V_{m}$ (hard 'Pyrex' glass) is greater than $V_{m}$ (soft soda glass), which in turn is greater than $V_{s}$ (metal). The close agreement between the present values with brass electrodes and those of Llewellyn Jones et al. clearly indicates that the above difference is not due to the use of a.c. Moreover, the larger values of $V_{m}$ with glass electrodes cannot be attributed to a difference

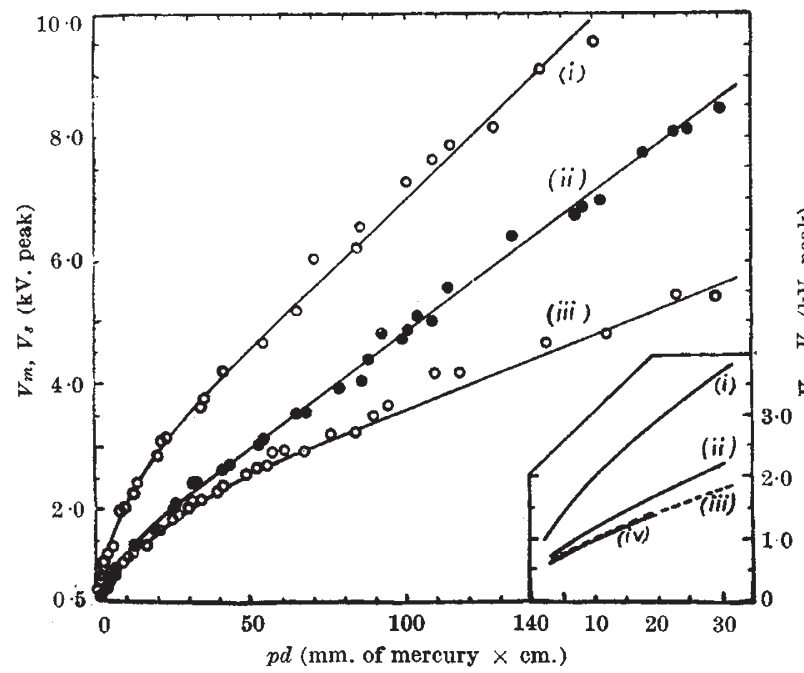

Fig. 1. Paschen's curves in air for various electrodes. (i) Hard 'Pyrex' glass electrodes; (ii) soft soda glass electrodes; (iii) brass electrodes ; (iv) copper electrodes (in inset), after Llewellyn (i), (ii) and (iii): under a.c. excitation; peak values are plotted in the work function of the electrodes, because differences in the breakdown potential for cathodes of different work function are a maximum near the minimum sparking potential and tend to become zero for higher pressures. In the present case, however, the difference $V_{m}-V_{8}$ is found to increase with $p \times d$. This difference may be attributed, at least in part, to a fall in potential on the glass electrodes depending on the resistivity, $p$, of the electrodes, which also changes in the sense that $\rho$ (hard 'Pyrex' glass) is greater than $\rho$ (soft soda glass) which is greater than $\rho$ (metal).

Details of this work will be published elsewhere.

A similar difference in the Geiger threshold for Maze counters with external cathodes and that for Geiger counters with internal cathodes has been reported ${ }^{3,4}$. The plateau for Maze counters is also found to be appreciably longer ${ }^{4}$. Such results may also be due $e^{5}$ to a corresponding fall in potential on the glass walls of the Maze counters.

D. P. JATAR

H. D. SHARMA

Department of Physics,

University of Saugar,

Saugor (M.P.),

India.

June 4.

${ }^{1}$ Joshi, S. S., Curr. Sci., 8, 548 (1939).

'Llewellyn Jones, F., and Davies, D. E., Proc. Phys, Soe., B, 64, 397 (1951).

"Aron, A., Z. Phys., 134, 622 (1953).

' Yasin, M., Ahmed, R., and Gill, P. S., Nature, 167, 647 (1951); Ind. J.'Phys., 25, 182 (1951)

5 Cockroft, A. L., and Valentine, J. M., J. Sci. Instr., 27, 262 (1950).

\section{A Compact Soil Perfusion Apparatus}

Lees and Quastel ${ }^{1}$ described an apparatus for the perfusion of soil samples which obviated the need for disturbing the soil during the experiment. Their design was later modified by Audus', who used a negative pressure for circulating the perfusate. This apparatus was rather fragile and required a consider. able amount of incubator space. A modified design, using the same principle as the Audus apparatus, has been used here successfully for twelve months and the alterations did not apparently affect the efficiency of the perfuser compared with the earlier models.

The apparatus consisted of a $500-\mathrm{ml}$. Florence flask (Fig. 1) in which the neck was replaced by the tube $D$ (15 cm. by $2.5 \mathrm{~cm}$. internal diameter). The bottom of the tube $D$ was constricted to prevent the soil column from falling into the flask. The side- arm $C$ was bent at right-angles and extended up to the same height as the flask. A constriction, 1-2 mm. $\approx$ in diameter, was made at the point of fusion $(B)$ to restrict the movement of liquid from $C$ into the flask $A$. When the diameter of the constriction at $B$ was greater than $2 \mathrm{~mm}$., air was drawn directly into the flask rather than into the tube $F$. The tubes $E$ and $F$ had an internal diameter of $7 \mathrm{~mm}$. and were fixed as shown in Fig. 1. The tube $F$ was sealed into the right-angle bend of the side-arm $C$, which was found empirically to allow the optimal flow of air and perfusate into the tube $F$. The tube $E$ led to a water pump and the air-flow was controlled by an adjustable clip. No constrictions in tubes $E$ or $F$ were found to be necessary. A piece of glass tuhing $(G)$ filled with cotton-wool was placed in the open 